\title{
Adaptación del Inventario de Esquemas sobre la Apariencia-Revisado (ASI-R) para Población de Habla Hispana
}

\author{
Adaptation of the Appearance Schemas Inventory (ASI-R) for Spanish Speaking \\ Population
}

Cecilia Silva ${ }^{1}$, Blanca Elizabeth Jiménez-Cruz, Javier Ramos Rosales, Ariana Hernández, Karla Brito, Alma Moreno

\section{Resumen}

Otorgar una importancia excesiva a la apariencia física puede convertirse en un factor de riesgo para desarrollar actitudes y conductas poco saludables, por lo que es necesario contar con un instrumento válido y confiable que la evalúe. El objetivo de este trabajo fue adaptar el Appearance Schemas Inventory (ASI-R) desarrollado por Cash, Melnyk \& Hrabosky (2004a) para población de habla hispana, para lo cual participaron 579 jóvenes con edades entre 17 y 28 años. Los resultados muestran que la consistencia interna del instrumento es adecuada $(\alpha=.920)$, lo mismo que la de sus dos factores, Importancia Autoevaluativa $(\alpha=.891)$ e Importancia Motivacional $(\alpha=.864)$, que en conjunto explican el $55.0 \%$ de la varianza. Se concluye que el instrumento adaptado es apropiado para evaluar a jóvenes mexicanos.

Palabras clave: apariencia física, autoesquemas de la apariencia, actitudes

\begin{abstract}
The importance given to the physical appearance may be a risk factor for unhealthy attitudes and behaviors, so it is necessary to have a valid and reliable instrument to assess it. The aim of this study was to adapt the Appearance Schemas Inventory (ASI-R) developed by Cash, Melnyk \& Hrabosky (2004a), for the Spanish speaking population. 579 young men and women aged between 17 and 28 years participated. The results show that the internal consistency of the instrument is adequate $(\alpha=.920)$, as did its two factors, Self-assessed Significance $(\alpha=.891)$ and Motivational Significance $(\alpha=.864)$, and together account for $55.0 \%$ of the variance. We conclude that the adapted instrument is appropriate for evaluating young Mexicans.
\end{abstract}

Keywords: physical appearance, appearance self-schemes, attitudes

\footnotetext{
${ }^{1}$ Facultad de Psicología, Edificio "D”, Cubículo 12, Universidad Nacional Autónoma de México, Av. Universidad 3004, Colonia Copilco-Universidad, Delegación Coyoacán, C.P. 04510, México D.F. csilva@posgrado.unam.mx, $52(55) 56222236$
} 


\section{Introducción}

Los esquemas cognitivos son estructuras que le permiten al individuo seleccionar, organizar y representar la información que le rodea. Un tipo particular de esquemas cognitivos son los autoesquemas, que se refieren al individuo mismo. Fueron definidos por Markus (1977) como las "generalizaciones cognitivas acerca de sí mismo, derivadas de experiencias pasadas, que organizan y guían el procesamiento de la información que hace referencia a sí mismo, contenida en las experiencias sociales del individuo" (p.64). El contenido de los auto-esquemas se refleja implícitamente en conductas, actitudes, creencias y supuestos de las personas.

Un auto-esquema es una organización de las representaciones de experiencia con sentido personal, constituido por los supuestos que tiene cada persona respecto del mundo y de sí mismo, tales como la personalidad, el rol sexual, el peso corporal, la forma corporal, la apariencia física, el ejercicio físico y la dieta, entre otros (Eddleston, Veiga, \& Powell, 2006; Hargreaves \& Tiggemann, 2002; Martínez, Reyes, \& Paredes, 2010; Kendzierski, 2007; Kendzierski, Sheffield, \& Morganstein, 2002; Reissing, Binik, Khalif, Cohen, \& Amsel, 2003).

Los esquemas de dominio específico, como el de apariencia física, determinan si se otorga o no importancia emocional a determinado atributo. De esta manera, las personas que poseen un autoesquema sobre la apariencia, es porque la consideran como parte esencial de su autoimagen y autoestima. Así, una persona esquemática para la apariencia física considerará ese atributo como auto-descriptivo e importante para su propia autodefinición y reaccionará de manera diferente ante estímulos relacionados con la apariencia física, en comparación con otra persona que no es esquemática para la apariencia física (Cash \& Labarge, 1996).

Cash, Melnyk y Hrabosky (2004a), plantean que la información recibida acerca de la apariencia física genera presión e idealización de ciertos atributos. Si la persona en quien recae esta presión es esquemática para la apariencia física, tenderá a evaluar constantemente su propia apariencia y si no satisface su ideal, experimentará emociones negativas y activará mecanismos cognitivos y conductuales dirigidos a satisfacer el auto-esquema, lo que en ocasiones puede resultar en prácticas nocivas. Debido a la utilidad clínica que representa la posibilidad de evaluar la importancia que las personas le dan a su apariencia física, Cash y Labarge (1996) desarrollaron el Inventario de Esquemas sobre la Apariencia (ASI-R, por sus siglas en inglés). Se trata de un instrumento que evalúa la presencia de auto-esquemas de apariencia física, los cuales determinan si los individuos experimentan preocupación por la apariencia física en relación con ciertas creencias sobre la importancia, el significado y la influencia de la apariencia física en la vida propia (Cash \& Labarge, 1996; Cash, Melnyk, \& Hrabosky, 2004a).

En una versión corregida, Cash, Melnyk y Hrabosky (2004a), el Appearance Schemas Inventory-Revised (ASI-R) quedó constituido por dos factores: el primero conformado por 12 reactivos, se denominó Importancia Autoevaluativa y examina la medida en la que los individuos se definen a sí mismos a través de su apariencia física, que influirá en sus experiencias sociales y emocionales. El segundo factor, Importancia Motivacional, quedó conformado por 8 reactivos y evalúa el grado en el que las personas se involucran en conductas para el cuidado y el manejo de su apariencia física. En un análisis de consistencia interna diferenciado por sexo, se obtuvieron altos valores de confiabilidad, tanto en la prueba total ( $\alpha=.88$ para las mujeres y $\alpha=.90$ para los hombres), como en las subescalas. En la subescala de Importancia Autoevaluativa se obtuvieron valores de $\alpha=.82$ para las mujeres y $\alpha=.84$ para los hombres, en tanto que en la subescala de Importancia Motivacional se obtuvieron valores de $\alpha=.90$ para las mujeres y $\alpha=.84$ en el caso de los hombres. Un análisis factorial de componentes principales con rotación oblicua arrojó correlaciones moderadas entre ambos factores, .51 para mujeres y .58 para hombres $(p<.001)$.

El ASI-R ha mostrado congruencia con cuestionarios como el Multidimensional BodySelf Relations Questionnaire (MBSRQ) (Smith \& Davenport, 2012), el Current Thoughts Scale 
(CTS), y el Body Image Ideals Questionnaire (BiQ) (Ip \& Jarry, 2008), entre otros.

Se ha utilizado en diversas investigaciones para estudiar los esquemas corporales con relación a las cogniciones distorsionadas sobre la imagen corporal (Cash, Melnyk, \& Hrabosky, 2004a), con la insatisfacción corporal (Cash, Phillips, Santos, \& Hrabosky, 2004b; Ip \& Jarry, 2008; Ledoux, Winterowd, Richardson, \& Clark, 2010), con la baja autoestima (Cash et al., 2004), con las conductas alimentarias no saludables (Cash, Melnyk, \& Hrabosky, 2004a; Cash, 2005), con la sintomatología depresiva (Moreira et al., 2010; Moreira \& Canavarro, 2012), con el pobre funcionamiento psicosocial (Cash, Melnyk \& Hrabosky, 2004a), y con el perfeccionismo (Ledoux, et al., 2010) por citar algunos.

A la fecha, no es de nuestro conocimiento ningún instrumento construido en países de habla hispana que evalúe los auto-esquemas de la apariencia física. Sin embargo, diversos estudios han mostrado una elevada prevalencia de insatisfacción corporal (Acosta \& Gil, 2003; Casillas-Estrella, Montaño-Castrejón, ReyesVelázquez, Bacardí-Gascón, \& Jiménez-Cruz, 2006; Vázquez, López, Álvarez, Mancilla, \& Oliva, 2006), y de propensión a los trastornos de la conducta alimentaria (Álvarez-Rayon et al., 2004; ENSANUT, 2012; Mancilla-Díaz et al., 2007). A su vez, en estudios realizados en otros países resulta claro que este dominio se asocia con los esquemas sobre la apariencia física (Cash, Melnyk, \& Hrabosky, 2004a; Cash, Phillips, Santos, \& Hrabosky, 2004b; Ledoux, Winterowd, Richardson, \& Clark, 2010; Smith \& Davenport, 2012). Por consiguiente se considera útil contar con la validación de un instrumento que evalúe dichos esquemas.

Ya que el ASI-R ha demostrado ser un instrumento útil en el contexto clínico y como herramienta de investigación en diversos estudios (Cash \& Grasso, 2005; Clark \& Tiggemann, 2007; Rusticus, Hubley, \& Zumbo, 2008), el objetivo del presente trabajo fue adaptarlo a la población mexicana, específicamente a jóvenes. La elección de ese grupo etario se debe a que se encuentran en una etapa de adaptación en diversos ámbitos (Gonçalvez \& Barrios, 2008) y a que constituye uno de los grupos poblacionales que presentan mayor riesgo de presentar conductas poco saludables relacionadas con la figura corporal, y en general con la apariencia física (Baile, Guillén, \& Garrido, 2002; Busaniche, Eymann, Mulli, Paz, \& Catsicaris, 2006; Chimenos et al., 2003; Lameiras, Calado, Rodríguez, \& Fernández, 2003; Rodríguez \& Cruz, 2008; Rodríguez, Goñi, \& Ruiz de Azúa, 2006).

\section{Método}

\section{Participantes}

Se obtuvo el consentimiento informado y la participación de 579 jóvenes $(51.3 \%$ hombres y $48.7 \%$ mujeres) con edades entre 17 y 28 años $(M=21.38, D E=2.34)$, los cuales fueron invitados a participar en diferentes escuelas de nivel medio superior de la Ciudad de México. El 0.4\% de los participantes contaban con escolaridad de secundaria, el $6.4 \%$ de bachillerato, el $90.3 \%$ de licenciatura y el $2.2 \%$ de posgrado. El IMC de los participantes, calculado a partir de su auto-reporte de peso y talla, se ubicó entre 15.43 y 44.29 $(M=23.40, \quad D E=3.54)$. La aplicación del instrumento fue conducida por los Psicólogos responsables de la investigación y fue realizada dentro de las instalaciones escolares donde fueron captados.

\section{Instrumentos}

1) Appearance Schemas Inventory-Revised (ASI-R) (Cash, Melnyk, \& Hrabosky, 2004a). Compuesto por 20 reactivos con 5 opciones de respuesta en una escala de tipo Likert, de acuerdo al orden $\mathrm{y}$ al número de respuestas del instrumento original: $1=$ totalmente de acuerdo, $2=e n$ desacuerdo, $3=$ ni de acuerdo ni en desacuerdo, 4=de acuerdo y 5=totalmente de acuerdo.

2) Prueba de actitudes hacia la alimentación (EAT-40, por sus siglas en inglés). Este instrumento se aplicó para probar la validez convergente del ASI-R. Los reactivos de este instrumento constituyen un conjunto de actitudes y conductas autopercibidas que son características de los trastornos de la conducta alimentaria, como el disgusto patológico del propio cuerpo y el deseo de cambiarlo por medio de la pérdida de peso, que puede incluir restricción alimentaria o 
purgas (Morry \& Staka, 2001). Al momento de su construcción (Garner \& Garfinkel, 1979) se obtuvo una confiabilidad de .79 para las mujeres anoréxicas y de .94 para la muestra total. Según su validación en población mexicana (ÁlvarezRayón et al., 2004), el instrumento evalúa cinco dimensiones: Restricción alimentaria $(\alpha=.88)$, Bulimia $(\alpha=.87)$, Impulso hacia la delgadez $(\alpha=.85)$, Preocupación por la comida $(\alpha=.77)$ y Presión social percibida $(\alpha=.76)$.

\section{Procedimiento}

La traducción y piloteo del cuestionario ASI$\mathrm{R}$ formaron parte de un trabajo de investigación (Jiménez-Cruz, 2014), en el cual se exploró la preocupación por la apariencia física de un grupo de madres. Para ello, se realizó la traducción del inglés al español y se sometió a jueceo de un grupo de especialistas expertos en el campo de la conducta alimentaria. Se verificó que los reactivos fueran claros y que su contenido se mantuviera conforme a la versión original en inglés y se decidió reducir las opciones de respuesta de 5 a 4 , a fin de eliminar la posibilidad de que las respuestas se concentraran en el punto intermedio y no señalaran ninguna tendencia.

A partir de dicha traducción, se realizó un piloteo con 29 mujeres con edades entre 24 y 58 años de edad $(M=38.2 ; D E=7.5)$; en esta aplicación se encontró que 12 de los 20 reactivos correlacionaron con valores bajos $(r<.30)$ con el total del instrumento. Debido a que la mayoría de los reactivos estaban planteados de manera negativa (por ejemplo, "Vestirme bien no es una prioridad para mí”), se consideró la posibilidad de que resultaran confusos para los participantes. Por ello se modificaron para presentarlos en sentido afirmativo (por ejemplo "Vestirme bien es una prioridad para mí") tras lo cual, se sometieron nuevamente a un jueceo entre expertos. Una vez finalizado se decidió conservar todos los reactivos excepto uno, porque al quitar la negación resultaba idéntico a otro de los reactivos. Una vez modificado el instrumento, se realizó un segundo piloteo con 46 mujeres de edades comprendidas entre 24 y 58 años $(M=39.8, D E=9.3)$. A partir de dicho piloteo, se eliminó un reactivo más por mostrar una correlación baja $(r=.08)$ con el puntaje de la sub-escala a la cual pertenecía.
Finalmente, después de dichos ajustes, el instrumento en su versión al español quedó conformado por 18 reactivos (Jiménez-Cruz, 2014).

Tras la aplicación a los 579 participantes, se analizaron las propiedades psicométricas del instrumento siguiendo el procedimiento sugerido por Nunnally y Bernstein, (1994). El análisis estadístico se realizó con el paquete estadístico SPSS versión 20.0; los análisis estadísticos que se llevaron a cabo fueron: análisis de frecuencias, $t$ de Student para muestras independientes, análisis factorial exploratorio, y obtención de la consistencia interna mediante el alfa de Cronbach, tanto para la totalidad del instrumento, como para cada una de sus dimensiones.

\section{Resultados}

\section{Análisis psicométrico}

El análisis de frecuencias inicial mostró una distribución normal en los 18 reactivos. A continuación se calculó la sumatoria de todos los reactivos y a partir del resultado de esta suma, se creó una nueva variable que organizara los puntajes en cuartiles. Tomando como grupos extremos el cuartil inferior y el cuartil superior se realizó un análisis mediante la prueba $t$ de Student para verificar el poder de discriminación de cada reactivo entre grupos extremos (Tabla 1). Todos los reactivos discriminaron, por lo que no se eliminó ninguno.

Posteriormente, se realizó un análisis factorial exploratorio con el método de extracción de componentes principales, obteniendo una correlación alta entre los factores $(r=.57, p<.05)$. En este primer análisis se obtuvo una medida de suficiencia muestral Kaiser-Meyer-Olkin (KMO) de $.94 \mathrm{y}$ un resultado significativo $(p<0.001)$ para la prueba de esfericidad de Bartlett, obteniendo 3 factores (Tabla 2), que en conjunto explican el $59.08 \%$ de varianza. El primer factor, explicó el $44.31 \%$, el segundo el $9.19 \%$ y el tercero el $5.57 \%$. Se conservaron únicamente los reactivos que obtuvieron una carga $\geq .40$, eliminándose así el reactivo 10. "Mi apariencia física ha tenido mucha influencia en mi vida".

Con los 17 reactivos restantes se condujo un segundo análisis factorial, obteniendo un índice 
Tabla 1. Pruebas $t$ de Student para diferencias entre grupos extremos

\begin{tabular}{|c|c|c|c|c|c|}
\hline \multirow[t]{2}{*}{ Reactivos } & \multirow[t]{2}{*}{$t$} & \multirow[t]{2}{*}{$g l$} & \multirow[t]{2}{*}{$p$} & \multirow{2}{*}{$\begin{array}{c}\text { Grupo } \\
\text { bajo }\end{array}$} & \multirow{2}{*}{$\begin{array}{l}\begin{array}{c}\text { Grupo } \\
\text { alto }\end{array} \\
M(D E)\end{array}$} \\
\hline & & & & & \\
\hline $\begin{array}{l}\text { 1. Empleo mucho tiempo ocupándome de mi } \\
\text { apariencia física. }\end{array}$ & -12.520 & 269 & $<.001$ & $\begin{array}{c}2.05 \\
(.739)\end{array}$ & $\begin{array}{c}3.13 \\
(.669)\end{array}$ \\
\hline $\begin{array}{l}\text { 2. Cuando encuentro gente que se ve bien, } \\
\text { me preguntó qué tan bien me veo yo. }\end{array}$ & -17.917 & 268.941 & $<.001$ & $\begin{array}{c}1.70 \\
(.765)\end{array}$ & $\begin{array}{l}3.27 \\
(.674)\end{array}$ \\
\hline 3. Trato de verme lo más bonita que puedo. & -12.410 & 267.424 & $<.001$ & $\begin{array}{c}2.24 \\
(.884)\end{array}$ & $\begin{array}{c}3.44 \\
(.711)\end{array}$ \\
\hline $\begin{array}{l}\text { 4. Presto mucha atención a la forma que me } \\
\text { veo. }\end{array}$ & -17.012 & 269 & $<.001$ & $\begin{array}{c}2.12 \\
(.712)\end{array}$ & $\begin{array}{c}3.47 \\
(.575)\end{array}$ \\
\hline $\begin{array}{l}\text { 5. Frecuentemente comparo mi apariencia } \\
\text { física con la de otras personas que veo. }\end{array}$ & -18.210 & 269 & $<.001$ & $\begin{array}{c}1.60 \\
(.730)\end{array}$ & $\begin{array}{c}3.25 \\
(.756)\end{array}$ \\
\hline $\begin{array}{l}\text { 6. Frecuentemente me veo en el espejo para } \\
\text { asegurarme de que me veo bien. }\end{array}$ & -18.275 & 269 & $<.001$ & $\begin{array}{c}1.97 \\
(.807)\end{array}$ & $\begin{array}{c}3.52 \\
(.533)\end{array}$ \\
\hline $\begin{array}{l}\text { 7. Cuando algo me hace sentir bien o mal al } \\
\text { respecto a mi apariencia física, tiendo a } \\
\text { pensar mucho en ello. }\end{array}$ & -22.472 & 269 & $<.001$ & $\begin{array}{c}1.57 \\
(.725)\end{array}$ & $\begin{array}{c}3.46 \\
(.653)\end{array}$ \\
\hline $\begin{array}{l}\text { 8. Si cierto día me gusta cómo me veo, } \\
\text { entonces es fácil sentirme feliz en otros } \\
\text { aspectos. }\end{array}$ & -15.970 & 250.534 & $<.001$ & $\begin{array}{c}2.10 \\
(.930)\end{array}$ & $\begin{array}{l}3.60 \\
(.608)\end{array}$ \\
\hline $\begin{array}{l}\text { 9. Me molestaría si alguien reaccionará } \\
\text { negativamente. }\end{array}$ & -12.883 & 269 & $<.001$ & $\begin{array}{c}1.76 \\
(.852)\end{array}$ & $\begin{array}{l}3.06 \\
(.807)\end{array}$ \\
\hline $\begin{array}{l}\text { 10. Mi apariencia física ha tenido mucha } \\
\text { influencia en mi vida. }\end{array}$ & -20.223 & 269 & $<.001$ & $\begin{array}{c}1.65 \\
(.712)\end{array}$ & $\begin{array}{c}3.33 \\
(.643)\end{array}$ \\
\hline 11. Vestirme bien es una prioridad para mí. & -15.110 & 269 & $<.001$ & $\begin{array}{c}1.88 \\
(.841)\end{array}$ & $\begin{array}{c}3.32 \\
(.712)\end{array}$ \\
\hline $\begin{array}{l}\text { 12. Cuando conozco por primera vez a una } \\
\text { persona, me pregunto qué piensa de } \\
\text { cómo me veo. }\end{array}$ & -19.031 & 234.721 & $<.001$ & $\begin{array}{l}1.43 \\
(.643)\end{array}$ & $\begin{array}{c}3.17 \\
(.827)\end{array}$ \\
\hline $\begin{array}{l}\text { 13. En mi vida diaria pasan muchas cosas } \\
\text { que me hacen pensar acerca de cómo } \\
\text { me veo. }\end{array}$ & -19.767 & 210.251 & $<.001$ & $\begin{array}{l}1.35 \\
(.521)\end{array}$ & $\begin{array}{c}2.99 \\
(.795)\end{array}$ \\
\hline $\begin{array}{l}\text { 14. Fantaseo acerca de cómo sería yo si mi } \\
\text { apariencia física fuera mejor. }\end{array}$ & -17.754 & 235.825 & $<.001$ & $\begin{array}{l}1.51 \\
(.688)\end{array}$ & $\begin{array}{c}3.23 \\
(.878)\end{array}$ \\
\hline $\begin{array}{l}\text { 15. Antes de salir de casa me aseguro que de } \\
\text { verme tan bien como puedo. }\end{array}$ & -14.370 & 269 & $<.001$ & $\begin{array}{c}2.03 \\
(.837)\end{array}$ & $\begin{array}{c}3.40 \\
(.705)\end{array}$ \\
\hline $\begin{array}{l}\text { 16. La forma en que me veo es una parte } \\
\text { importante de la persona que soy. }\end{array}$ & -15.267 & 269 & $<.001$ & $\begin{array}{c}1.98 \\
(.878)\end{array}$ & $\begin{array}{c}3.42 \\
(.637)\end{array}$ \\
\hline $\begin{array}{l}\text { 17. Controlando mi apariencia física puedo } \\
\text { controlar muchos de los eventos } \\
\text { sociales y emocionales de mi vida. }\end{array}$ & -20.785 & 269 & $<.001$ & $\begin{array}{l}1.49 \\
(.636)\end{array}$ & $\begin{array}{c}3.22 \\
(.736)\end{array}$ \\
\hline $\begin{array}{l}\text { 18. Mi apariencia física es la responsable de } \\
\text { mucho de lo que me ha pasado en la } \\
\text { vida. }\end{array}$ & -16.837 & 225.311 & $<.001$ & $\begin{array}{c}1.43 \\
(.632)\end{array}$ & $\begin{array}{c}3.00 \\
(.867)\end{array}$ \\
\hline
\end{tabular}


Tabla 2. Matriz de configuración (cargas factoriales) y comunalidades

\begin{tabular}{|c|c|c|c|}
\hline Reactivo & Factor 1 & Factor 2 & Comunalidades \\
\hline $\begin{array}{l}\text { 13. En mi vida diaria pasan muchas cosas que me } \\
\text { hacen pensar acerca de cómo me veo. }\end{array}$ & .833 & -.027 & 670 \\
\hline $\begin{array}{l}\text { 5. Frecuentemente comparo mi apariencia física con la } \\
\text { de otras personas que veo. }\end{array}$ & .795 & -.026 & .610 \\
\hline $\begin{array}{l}\text { 2. Cuando encuentro gente que se ve bien, me } \\
\text { preguntó qué tan bien me veo yo. }\end{array}$ & .688 & .085 & .548 \\
\hline $\begin{array}{l}\text { 7. Cuando algo me hace sentir bien o mal al respecto a } \\
\text { mi apariencia física, tiendo a pensar mucho en } \\
\text { ello. }\end{array}$ & .686 & .116 & .575 \\
\hline $\begin{array}{l}\text { 14. Fantaseo acerca de cómo sería yo si mi apariencia } \\
\text { física fuera mejor. }\end{array}$ & .788 & -.038 & .588 \\
\hline $\begin{array}{l}\text { 9. Me molestaría si alguien reaccionará negativamente } \\
\text { hacia mi apariencia física. }\end{array}$ & .726 & -.135 & .434 \\
\hline $\begin{array}{l}\text { 12. Cuando conozco por primera vez a una persona, } \\
\text { me pregunto qué piensa de cómo me veo. }\end{array}$ & .654 & .103 & .515 \\
\hline $\begin{array}{l}\text { 17. Controlando mi apariencia física puedo controlar } \\
\text { muchos de los eventos sociales y emocionales de } \\
\text { mi vida. }\end{array}$ & .624 & .166 & .535 \\
\hline $\begin{array}{l}\text { 18. Mi apariencia física es la responsable de mucho de } \\
\text { lo que me ha pasado en la vida. }\end{array}$ & .631 & .055 & .440 \\
\hline 3. Trato de verme lo más bonita que puedo. & -.106 & .792 & .543 \\
\hline 4. Presto mucha atención a la forma que me veo. & .053 & .787 & 670 \\
\hline $\begin{array}{l}\text { 1. Empleo mucho tiempo ocupándome de mi } \\
\text { apariencia física. }\end{array}$ & -.060 & .768 & .540 \\
\hline $\begin{array}{l}\text { 15. Antes de salir de casa me aseguro que de verme } \\
\text { tan bien como puedo. }\end{array}$ & .018 & .752 & .582 \\
\hline $\begin{array}{l}\text { 6. Frecuentemente me veo en el espejo para } \\
\text { asegurarme de que me veo bien. }\end{array}$ & .099 & .693 & .568 \\
\hline 11. Vestirme bien es una prioridad para mí. & .065 & .671 & .503 \\
\hline $\begin{array}{l}\text { 16. La forma en que me veo es una parte importante de } \\
\text { la persona que soy. }\end{array}$ & .170 & .583 & .481 \\
\hline
\end{tabular}

Tabla 3. Pruebas t entre sexos para los puntajes en escala total y en los factores

\begin{tabular}{|c|c|c|c|c|c|c|c|c|c|c|}
\hline \multirow{2}{*}{ Puntajes promediados } & \multicolumn{2}{|c|}{ Mujeres } & \multicolumn{2}{|c|}{ Hombres } & & \multirow[b]{2}{*}{$g l$} & \multirow[b]{2}{*}{$p$} & \multicolumn{2}{|c|}{$95 \% \mathrm{IC}$} & \multirow{2}{*}{$\begin{array}{l}d \text { de } \\
\text { Cohen }\end{array}$} \\
\hline & $M$ & $D E$ & $M$ & $D E$ & & & & LI & LS & \\
\hline $\begin{array}{l}\text { Preocupac } \\
\text { (total) }\end{array}$ & 2.89 & 0.63 & 2.68 & 0.68 & 1 & 546 & 1 & .09 & .31 & 0.32 \\
\hline Importancia Mo & 2.7 & 0.5 & 2.56 &  & & & & .07 & .28 & \\
\hline Importancia Autoevaluativa & 2.38 & 0.60 & 2.22 & 0.68 & 2.63 & 558 & $<.001$ & .04 & .26 & 0.24 \\
\hline
\end{tabular}

KMO de .93 y un resultado significativo $(p<0.001)$ para la prueba de esfericidad de Bartlett. En este segundo momento otro reactivo resultó con un valor de extracción menor a .40 ("Si cierto día me gusta cómo me veo, entonces es fácil sentirme feliz en otros aspectos"), por lo que se eliminó del análisis.
En un tercer análisis factorial con rotación oblicua mediante el método Oblimin directo, se volvió a obtener un índice KMO de .937 y un resultado significativo $(p<0.001)$ para la prueba de esfericidad de Bartlett. Se obtuvieron dos factores (Tabla 2), que en conjunto explicaron el $55.00 \%$ de la varianza. El primer factor, explicó el $44.64 \%$ 
de la varianza y quedó conformado por los reactivos que de acuerdo con Cash, Melnyk y Hrabosky (2004a) hacen referencia a la "Importancia Autoevaluativa" de la apariencia física, por lo cual se denominó de la misma manera. El segundo factor explicó el 10.36\% de la varianza y quedó conformado por los reactivos que de acuerdo con los autores del instrumento, hacen referencia a la "Importancia Motivacional" de la apariencia física, conservándose también su denominación.

Posteriormente, se condujo un análisis de la consistencia interna del instrumento, tanto para el puntaje total, como para cada una de las subescalas. Se obtuvo un $\alpha$ de Cronbach de .920 para la escala total, de $\alpha=.891$ para el factor "Importancia Autoevaluativa" y de $\alpha=.864$ para el factor "Importancia Motivacional". Asimismo, se observó que todas las correlaciones ítem-total fueron $\geq .494$. El instrumento final quedó conformado por 16 reactivos.

Por último, se exploró si existían diferencias entre hombres y mujeres llevando a cabo un análisis con pruebas $t$ para muestras independientes tanto con el puntaje total, como con el puntaje de los factores. Debido a que el factor de Importancia Autoevaluativa se conformó por nueve reactivos y el de Importancia Motivacional por siete, se obtuvieron los puntajes promedio de uno y otro factor, así como el puntaje promedio del total de la escala. Con esos puntajes se realizaron las pruebas $t$ con un ajuste de Bonferroni fijándose en $p \leq .016$; los resultados de las pruebas indicaron diferencias significativas entre hombres y mujeres, tanto en el puntaje total, como en los puntajes de cada uno de los factores (Tabla 3).

Se realizó un análisis de validación por convergencia utilizando el EAT-40, en tanto que la preocupación por la apariencia física y las actitudes hacia el peso y la alimentación se han asociado en otros estudios (Cash, Melnyk, \& Hrabosky, 2004a; Cash, 2005). El índice de correlación más elevado se encontró entre los factores del ASI-R y la motivación para adelgazar, pero también se asociaron significativamente con la restricción alimentaria, bulimia, preocupación por la comida y presión social percibida para ser delgados. Los resultados de las correlaciones se muestran en la Tabla 4. La asociación entre los puntajes totales de ambos instrumentos fue de $r=.457, p<.001$.

Tabla 4. Coeficientes de correlación de Pearson entre los factores del ASI-R y los factores del EAT-40

\begin{tabular}{lcc}
\hline \multicolumn{1}{c}{ Factores del } & \multicolumn{2}{c}{ Factores del ASI-R } \\
\cline { 2 - 3 } & $\begin{array}{c}\text { Importancia } \\
\text { autoevaluativa }\end{array}$ & $\begin{array}{c}\text { Importancia } \\
\text { motivacional }\end{array}$ \\
\hline $\begin{array}{l}\text { Dieta restrictiva } \\
\text { Bulimia }\end{array}$ & $.296 * * *$ & $.242 * * *$ \\
$\begin{array}{l}\text { Motivación para } \\
\text { adelgazar }\end{array}$ & $.260 * * *$ & $.202 * * *$ \\
$\begin{array}{l}\text { Preocupación por la } \\
\text { comida }\end{array}$ & $.306 * * *$ & $.365^{* * *}$ \\
$\begin{array}{l}\text { Presión social } \\
\text { percibida }\end{array}$ & $.180 * * *$ & $.123 * *$ \\
$* * * p<.001 ; * * p<.01$ & & \\
\hline
\end{tabular}

\section{Discusión}

Este estudio se generó en razón de la necesidad de contar con un instrumento confiable y válido para evaluar la preocupación de los jóvenes por su apariencia física emanada de sus auto-esquemas. Asimismo considerando que el ASI-R ha mostrado en otras poblaciones buen funcionamiento para este propósito (Cash, Melnyk, \& Hrabosky, 2004a; Ip \& Jarry, 2008; Ledoux et al., 2010; Smith \& Davenport, 2012) se planteó el objetivo de adaptarlo para población mexicana.

Los datos obtenidos arrojaron una organización de los reactivos muy similar a la obtenida por Cash, Melnyk, \& Hrabosky (2004a), ya que de igual manera se agruparon en dos factores, el primero sobre la Importancia Autoevaluativa, y el segundo sobre la Importancia Motivacional que. El primer factor se asocia, de acuerdo con la literatura científica sobre el tema, con actitudes negativas hacia el peso y la alimentación (Cash, 2005; Cash, Phillips, Santos, \& Hrabosky, 2004b; Ip \& Jarry, 2008; Ledoux et al., 2010), mientras que el segundo pareciera estar más relacionado con una preocupación normal por la apariencia física (Cash, Melnyk, \& Hrabosky, 2004a; Jiménez-Cruz, 2014). De acuerdo con los resultados de este trabajo, existe una correlación moderada entre ambos factores, de tal manera que 
las personas que consideran que su apariencia física es importante, tienden a mostrar un mayor número de conductas relacionadas con el cuidado de su apariencia física. Dicho de otro modo, quienes muestren actitudes negativas hacia su figura corporal, tienden a pensar que su apariencia es de gran relevancia.

A pesar de que los reactivos se agruparon en los dos factores originales, en el de Importancia Autoevaluativa hubieron algunos cambios, pues algunos de los reactivos que originalmente conformaban el instrumento, obtuvieron cargas $\leq$ .40. En cambio, el factor Importancia Motivacional no sufrió ninguna modificación.

De acuerdo con estudios realizados con la versión en inglés del ASI-R (Cash, Melnyk, \& Hrabosky, 2004a; Cash, 2005), en la validación por convergencia que se realizó con la adaptación para población de habla hispana utilizando como referencia el EAT-40, se encontraron asociaciones significativas que indican que el instrumento conserva sus propiedades originales de validez.

A partir de los resultados de este estudio, se concluye que la adaptación del ASI-R para jóvenes mexicanos cuenta con características psicométricas adecuadas. Esto es, la distribución de los datos es normal, la consistencia interna es elevada - tanto en el puntaje total del instrumento, como en cada una de sus sub-escalas -, y cada uno de los factores se agrupa de manera coherente, de acuerdo a la teoría que le subyace (Cash \& Labarge, 1996).

Con el uso del ASI-R adaptado, se podrá explorar la relación de los esquemas corporales con variables referentes a la sintomatología propia de los trastornos alimentarios, depresión, funcionamiento psicosocial, actitudes hacia la alimentación, estigmatización de la obesidad y el cuidado de la salud, entre otras. Por lo consiguiente se lo considera un instrumento útil tanto para la práctica clínica como para la obtención de datos en proyectos de investigación. En estudios posteriores, sería recomendable extender la validación a población hispana en general.

\section{Referencias}

Acosta, G. M., \& Gómez, P. G. (2003). Insatisfacción corporal y seguimiento de dieta. Una comparación transcultural entre adolescentes de España y México. International Journal of Clinical and Health Psychology, 3(1), 9-21.

Álvarez-Rayón, G., Mancilla Díaz, J. M., Vázquez Arévalo, R., Unikel-Santoncini, C., Cabellero-Romo, A., \& Mercado-Corona, D. (2004). Validity of the Eating Attitudes Test: A study of Mexican eating disorders patients. Eating and Weight Disorders, 4, 243-248.

Baile, A. J., Guillén, G. F., \& Garrido, L. E. (2002). Insatisfacción corporal en adolescentes medida con el Body Shape Questionnaire (BSQ): Efecto del anonimato, el sexo y la edad. Revista Internacional de Psicología Clínica y de la Salud, 2(3), 439450.

Busaniche, J., Eymann, A., Mulli, V., Paz, M., \& Catsicaris, C. (2006). Asociación entre adolescentes tatuados y conductas de riesgo. Archivos Argentinos de Pediatría, 104(4), 309-315.

Cash, T. F., \& Labarge, A. S. (1996). Development of the Appearance Schemas Inventory: A new cognitive body-image assessment. Cognitive therapy and Research, 20(1), 37-50.

Cash, T. F., Melnyk, S. E., \& Hrabosky, J. I. (2004). The assessment of body image investment: An extensive revision of the Appearance Schemas Inventory. International Journal of Eating Disorders, 35(3), 305-316.

Cash, T., Phillips, K., Santos, M., \& Hrabosky, J. (2004). Measuring 'negative body image': Validation of the Body Image Disturbance Questionnaire in a nonclinical population. Body Image, 1(4), 363-372. http://dx.doi.org/10.1016/j.bodyim.2004.10.0 01

Cash, T. (2005). The influence of sociocultural factors on body image: Searching for constructs. Clinical Psychology: Science and 
Practice, 12(4), 438-442. doi: 10.1093/clipsy.bpi055.

Casillas-Estrella, M., Montaño, Castrejón, N., Reyes-Velázquez, V., Bacardí-Gascón, M., \& Jiménez Cruz, A. (2006). A mayor IMC mayor grado de insatisfacción de la imagen corporal. Revista Biomédica, 17, 243-249.

Chimeos, K. E., Batlle, T. I., Velásquez, R. S., García, C. T., Viñals, I. H., \& Roselló, L. X. (2003). Estética y cultura: Patología bucal asociada a ciertas modas "actuales". Medicina Oral, 8(3), 197-206.

Eddleston, K. A., Veiga, J. F., \& Powell, G. N. (2006). Explaining sex differences in managerial career satisfier preferences: The role of gender self-schema. Journal of Applied Psychology, 91(2), 437.

Garner, D., \& Garfinkel, P. (1979). The Eating Attitudes Test: An index of the symptoms of anorexia nervosa. Psychological Medicine, 9, 273-279.

Gonçalvez, M. I., \& Barrios, L. (2008). Inventário de preocupações e valorizações para jovens: Contributo para a avaliação das atribuições de preocupação e importancia a áreas da vida de jovens na fase de transição para a adultícia. Revista Iberoamericana de Diagnostico y Evaluación - e Avaliação Psicológica, 26(2), 141-166.

Hargreaves, D., \& Tiggemann, M. (2002). The effect of television commercials on mood and body dissatisfaction: The role of appearance-schema activation. Journal of Social and Clinical Psychology, 21(3), 287308.

Ip, K., \& Jarry, J. L. (2008). Investment in body image for self-definition results in greater vulnerability to the thin media than does investment in appearance management. Body Image, 5, 59-69. doi: 10.1016/j.bodyim.2007.08.002.

http://dx.doi.org/10.1016/j.bodyim.2007.08.0 02Kendzierski, D. (2007).

Jiménez, B. E. (2014). Obesidad: Estigmatización y preocupación por la apariencia física. (Tesis doctoral). Universidad Nacional Autónoma de México, Distrito Federal, México.
Kendzierski, D., Sheffield, A., \& Morganstein, M. S. (2002). The role of self-schema in attributions for own versus other's exercise lapse. Basic and applied social psychology, 24(4), 251-260.

Lameiras, F. M., Calado, O. M., Rodríguez, C. Y., \& Fernández, P. M. (2003). Hábitos alimentarios e imagen corporal en estudiantes universitarios sin trastornos alimentarios. Revista Internacional de Psicología Clínica y de la Salud, 3(1), 2333.

Ledoux, T., Winterowd, C., Richardson, T., \& Clark, D. J. (2010). Relationship of negative self schemas and attachment styles with appearance schemas. Body Image, 7(3), 213217. doi: 10.1016/j.bodyim.2010.02.003.

Mancilla-Díaz, J., Franco-Paredes, K., VázquezArevalo, R., López-Aguilar, X., ÁlvarezRayón, G., \& Tellez-Giron, M. (2007). A Two-Stage Epidemiologic Study on Prevalence of Eating Disorders in Female University Students in Mexico. European Eating Disorders Review, 15, 463-470.

Martínez, I., Reyes, I., Paredes, R. G. (2010). Autoesquema sexual femenino: Construcción y validación de una escala para población mexicana. Revista Iberoamericana de diagnostico y Evaluación - e Avaliação Psicológica, 30(2), 143-155.

Melnyk, S. E., Cash, T. F., \& Janda, L. (2004). Body image ups and downs: Prediction of intra-individual level and variability of women's daily body image experiences. Body Image, 1(3), 225-235.

Moreira, H., \& Canavarro, M. C. (2012). The association between self-consciousness about appearance and psychological adjustment among newly diagnosed breast cancer patients and survivors: The moderating role of appearance investment. Body Image, 9, 209-215. doi: 10.1016/j.bodyim.2011.11.003.

Moreira, H., Silva, S., \& Canavarro, M. C. (2010). The role of appearance investment in the adjustment of women with breast cancer. Psycho-Oncology, 19, 956-966. doi: 10.1002/pon.1647. http:// dx.doi.org/10.1002/pon.1647 
Morry, M., \& Staka, S. (2001). Magazine exposure: Internalization, selfobjetification, eating attitudes, and body satisfaction in male and female university students. Canadian Journal of Behavioural Science, 33, 269-279.

Nunnally, J.C, \& Bernstein, Y.J. (1994). Psychometric theory (Tercera edición). New York: McGraw Hill.

Reissing, E. D., Binik, Y. M., Khalif, S., Cohen, D., \& Amsel, R. (2003). Etiological correlates of vaginismus: Sexual and physical abuse, sexual knowledge, sexual self-schema, and relationship adjustment. Journal of Sex \& Marital Therapy, 29(1), 47-59.

Rodríguez, S., \& Cruz, S. (2008). Insatisfacción corporal en adolescentes latinoamericanas y españolas. Psicothema, 20(1), 131-137.

Rodríguez, A., Goñi, A., \& Ruíz de Azúa, S. (2006). Autoconcepto físico y estilos de vida en la adolescencia. Intervención Psicosocial, 15(1), 81-94.

Rudiger, J. A., Cash, T. F., Roehrig, M., \& Thompson, J. K. (2007). Day-to-day body image states: Prospective predictors of intraindividual level and variability. Body Image, $\quad 4(1), \quad 1-9 . \quad$ doi: 10.1016/j.bodyim.2006.11.004.

Smith, A. R., \& Davenport, B. R. (2012). An evaluation of body Image assessments in hispanic college women: The Multidimensional Body-Self Relations Questionnaire and the Appearance Schemas Inventory-Revised. Journal of College Counseling, 15, 198-214.

Vázquez, A. R., López, A. X., Álvarez, R. G., Mancilla, D. J., \& Oliva, R. A. (2006). Insatisfacción corporal e influencia de los modelos estéticos en niños y jóvenes varones mexicanos. Enseñanza e Investigación en Psicología, 11(1), 185-197. 